\title{
THE GLOBULAR CLUSTER SYSTEM OF M87
}

\author{
J.G. COHEN
}

Palomar Observatory, MS 105-24

California Institute of Technology Pasadena, CA 91125, USA

We have begun a program with the Low Resolution Imaging Spectrograph, operating with 30 multi-slits per exposure (Oke et al 1994, Cohen et al 1993) on the ten-meter W. M. Keck telescope located on Mauna Kea, Hawaii to observe the globular cluster systems of the Virgo ellipticals. We expect to learn about the dark matter content of galaxy halos, the formation of these halos and their cluster systems, possibly the formation of the galaxy's themselves, the interaction of the cluster system with the galaxy's gravitational potential (i.e. rotation and spinup), and the homogeneity of chemical evolution in various places. The metallicity of the globular clusters versus the metallicity of the underlying galaxy can also by analyzed. In addition the dynamics (i.e. in practice, the rotation and velocity dispersion) of the halo of the parent galaxy versus the globular cluster system can be determined.

Although work has commenced on these Virgo systems (Mould, Oke \& Nemec 1987, Mould et al 1990, Huchra \& Brodie 1987 and Brodie \& Huchra 1991), the sample observed to date is extremely limited. The multi-slit mode of the LRIS together with the large aperture of the Keck telescope should make it possible to rapidly increase this sample and significantly improve the kinematic analysis. At the present time, 6 fields around M87 have been observed with LRIS using the multi-slit mode during March, 1994. The slit-masks were designed using the list of candidate globular clusters around M87 of Strom et al (1981). Each field was observed for 3000 seconds using the 600 line grating centered at $8100 \mathrm{~A}$. This gives 1.24 $\AA /$ pixel, and $3.7 \AA /$ resolution element. The spectra cover the region of the infrared Ca triplet. The detector is a 2048 x 2048 Tektronix CCD read out using 2 amplifiers. The detector noise level was excessively high (readout noise 15-20 electrons rms, and even worse when pickup problems occured) until June, 1994 due to problems in the LRIS CCD electronics. The detector readout noise at the present time is 7 electrons rms. The time now required 
to achieve alignment is 15 minutes.

At this time only the 13 brightest of the $\approx 75$ spectra on three of the slit-masks have been analyzed. One object (Strom 982) turned out to be a background galaxy, while 6 have radial velocities of $0 \pm 100 \mathrm{~km} \mathrm{sec}^{-1}$ and are assumed to be foreground galactic stars. (Objects number 417, 1093, $1155,1200,1370$, and 1391 from Strom's list turned out to be genuine M87 globular clusters, while objects $354,782,1086,1216$, and 1341 appear to be foreground galactic stars.) Prior to being chosen for spectroscopy, the objects were all checked on the available LRIS direct images (scale 4.67 arc-sec/pixel) to separate stars from galaxies; roughly $20 \%$ of the objects in Strom et al's sample of globular clusters in the periphery of M87 have image morphologies inconsistent with their being globular clusters around M87, with most being obvious background galaxies.

As a check, we observed $3 \mathrm{M} 87$ globular clusters in common with earlier work. The two in common with Mould et al's sample showed good agreement. There is one object in common with the Huchra \& Brodie sample, but the agreement there is very poor, with a difference of over $-400 \mathrm{~km}$ $\sec ^{-1}$.

Armandroff \& Zinn (1988) observed the infrared Ca triplet in the integrated light of galactic globular clusters. We use their definition of the wavelength intervals and their relationship between $\Sigma\left(W_{\lambda}\right)$ (the sum of the equivalent widths of the 3 lines comprising the $\mathrm{Ca}$ triplet) and $[\mathrm{Fe} / \mathrm{H}]$ to estimate abundances for our 6 genuine M87 globular clusters. Four of them are very metal rich, near or above solar metallicity, while the other two have $[\mathrm{Fe} / \mathrm{H}]=-1.7 \pm 0.1 \mathrm{dex}$.

\section{References}

Armandroff, T.E. \& Zinn, R. 1988 A.J., 96, 92

Brodie, J.P. \& Huchra, J.P. 1991, Ap.J., 379, 157

Cohen, J.G., Cromer, J.L., Southard, S.Jr., \& Clowe, D. in Third Annual Conference on Astronomical Data Analysis Software and Systems, 1993, PASP Conference Proceedings

Huchra, J.P. \& Brodie J.P. 1987, A.J., 93, 779

Mould, J.R., Oke, J.B. \& Nemec, J.M. 1987, A.J., 93, 53

Mould, J.R., Oke, J.B., de Zeeuw, P.T., \& Nemec, J.M. 1990, A.J., 99, 1823

J.B.Oke, J.G.Cohen, M.Carr, A.Dingizian, F.Harris, R.Lucinio, S.Labrecque, W.Schaal \& S.Southard Jr., Proc. SPIE, 2198, 178, 1994

Strom, S.E., Forte, J., Harris, W., Strom, K.M., Wells, D., \& Smith, M. 1981, Ap.J., 245, 416 